\title{
Plasticity in cancer cell populations: biology, mathematics and philosophy of cancer ${ }^{\star}$
}

\author{
Jean Clairambault ${ }^{1[0000-0002-8336-9641]}$ \\ Inria \& LJLL, Sorbonne University, Paris, France \\ jean.clairambault@inria.fr \\ https://who.rocq.inria.fr/Jean.Clairambault/Jean_Clairambault_en.html
}

\begin{abstract}
In this presentation that partly subsumes and summarises in the form of adapted excerpts some recent articles of which I am author or co-author [2], [3], [11], I suggest that cancer is fundamentally a disease of the control of cell differentiation in multicellular organisms, uncontrolled cell proliferation being a mere consequence of blockade, or unbalance, of cell differentiations. Cancer cell populations, that can reverse the sense of differentiations, are extremely plastic and able to adapt without mutations their phenotypes in order to transiently resist drug insults [10], which is likely due to the reactivation of ancient, normally silenced, genes [5], [7], [12]. Stepping from mathematical models of non genetic plasticity in cancer cell populations [4], [6] and questions they raise, I propose an evolutionary biology approach to shed light on this problem a) from a theoretical viewpoint by a description of multicellular organisms in terms of multi-level structures, which integrate function and matter from lower to upper levels, and b) from a practical point of view by proposing future tracks for cancer therapeutics, as cancer is primarily a failure of multicellularity in animals and humans. This approach resorts to the emergent field of knowledge known as philosophy of cancer [1], [8], [9].
\end{abstract}

Keywords: Cancer Cells $\cdot$ Plasticity $\cdot$ Mathematical models $\cdot$ Philosophy of cancer

\section{Introduction}

Coherent multicellular organisms are not only cohesive from a spatial, anatomical point of view, but also coherent from the phenotypic and cell-functional point of view of compatibility, cooperativity (division of tasks) between cells and tissues, making possible the achievement of a stable, functional and reproductive whole.

I make here the simple hypothesis of a system of communication ways between trees of differentiation, relying on the control of transcription factors that determine differentiations, and that I call "the cohesion watch". It may be considered as a part of the immune system, whose armed force is the immune response,

\footnotetext{
* Invited paper
} 
innate as well as adaptive, humoral and cellular, but is not the whole of the immune system, that I view as the conductor of the unity of the organism. Within the immune system in this extended vision that is thus more general than the immune response, this "cohesion watch" is in charge of the control of compatibilities and cooperations between the anatomical and the phenotypic/cell-functional systems, ultimately leading to an anatomically cohesive and functionally coherent multicellular organism.

\section{Plasticity in cancer cell populations}

\subsection{Non genetic phenotype switching}

Phenotype switching, or more generally continuous phenotype-determined cell plasticity, is an essential process originally observed during development, but is also now recognised as an important phenomenon upon injury and disease. One of the best described examples of phenotypic switching in cancer depends on the process of epithelial to mesenchymal transition (EMT) and its reversion (MET) [11]. In the context of anti-cancer therapies, cell plasticity enables tumour cells to change to a cell phenotypic identity that may be dependent or not on the drug target, without additional secondary genetic mutations. Indeed, the discovery of oncogenic-driven mutations favoured the development of diverse targeted therapies and showed unprecedented clinical response. Unfortunately, responses are in general incomplete and transient, as resistances develop upon continuous treatment exposure. Along with well-known genetic alterations, cell plasticity has recently emerged as an unavoidable contributor to therapy evasion.

\subsection{Transient drug-induced tolerance in cancer}

The biological mechanisms that induce cancer cell plasticity upon drug treatment remain to be fully established. Nonetheless, they seem to involve a transition whereby tumour cells undergo a slow proliferating drug tolerant state, nailed the name of drug-tolerant persisters (DTPs), before possibly further developing secondary mutational drug resistance. Persisters were firstly described in bacteria upon antibiotic challenges. Similarly, a sub-population of non-small cell lung cancer (NSCLC) cells has been shown to engage in a reversible phenotypic change in which DTPs survive the initial onslaught of anti-cancer therapies [10]. Similar phenomena were observed in glioblastoma and melanoma. Biological observations support the idea that a tiny subpopulation, diverging from predominant cell phenotype prior to targeted therapy, may be subjected to a combination of Lamarckian plasticity and Darwinian selection upon anti-cancer therapies.

\subsection{Dedifferentiation and transdifferentiation}

Conversion of lineage has been extensively studied in the context of development. The well-known, metaphoric, Waddington landscape has been proposed 
to illustrate the fact that a progenitor cell normally rolls down within epigenetic differentiation valleys and can develop, due to phenotypic bifurcations, into the various terminally differentiated tissue types that constitute a coherent multicellular organism (see also Section 3). In the context of cancer, dedifferentiation and transdifferentiation have been observed upon therapeutic challenges, which suggests a possible plasticity in the cancers Waddington landscape, with metaphorically flattened valleys and lowered epigenetic barriers.

\section{Mathematics of plasticity with therapeutic control}

\subsection{How to mathematically model plasticity in cancer}

Cell plasticity is the ability of cells to change their phenotypes without genetic mutations in response to environmental cues. In a series of papers starting in 2013, a team of mathematicians, to which I belong, at Laboratoire JacquesLouis Lions, Sorbonne University, Paris, taking plasticity to be a concept of the continuum, and initially stimulated by an article published in 2010 [10] that reported reversible resistance in a cancer cell culture exposed to massive doses of drugs, gradually and completely reversed when the drug was withdrawn from the culture, tackled the question of understanding and predicting this plastic behaviour of cancer cell populations by mathematical modelling (reviewed in [4]). The behaviour of these plastic cell populations was modelled by partial differential equations (PDEs) in which the structuring variable, i.e., the parameter-like one that codes for the biological variability of interest, i.e., the heterogeneity of cell population, was here a continuous variable representing the expression of a continuous resistance phenotype, from 0 (totally sensitive) to 1 (totally resistant). The modelled cell populations are able to change their phenotypes under high drug pressure so as to become reversibly resistant to the drug, meaning by this the fact that, as real cells when the drug was rinsed from the culture, the cell population spontaneously became sensitive again to the same drug [10].

\subsection{Adaptive dynamics: asymptotic behaviour of cell populations}

Such mathematical models, that resort to the field of adaptive dynamics [4], [6] consist of partial differential equations (PDEs) structured in continuous phenotypes coding for the expression of drug resistance genes; they involve different functions representing targets for different drugs, cytotoxic and cytostatic, with complementary effects in limiting tumour growth. These phenotypes evolve continuously under drug exposure, and their fate governs the evolution of the cell population under treatment.

This evolutionary point of view, which relies on biological observations and resulting modelling assumptions, naturally extends to questioning the very nature of cancer as evolutionary disease, seen not only at the short time scale of a human life, but also at the billion year-long time scale of Darwinian evolution, from unicellular organisms to evolved multicellular organs such as animals and man. Such questioning, not so recent, but recently revived [5], [12], [7] in cancer studies, may have consequences for understanding and treating cancer 


\subsection{Theoretical therapeutics: multi-targeted optimal control}

These mathematical models, intended to represent the effects of a cancer treatment on cell populations, and ultimately on patients, with the aim to overcome their capacities of resistance induced by the treatment itself, naturally gave later rise to the proposal of theoretically optimised therapeutic strategies. Methods of optimal control have been used, taking inevitable emergence of drug resistance into account, to achieve the best strategies to contain the expansion of a tumour. Such strategies, that have recently been the object of active research aim at containing or eradicating cancer growth, avoiding the two major pitfalls of treatments in clinical oncology, namely unwanted toxic side effects in healthy cell populations and emergence of resistance in cancer populations [4], [6].

\section{Evolutionary biology and philosophy of cancer}

\section{1 'Nothing makes sense in biology except in the light of evolution'}

This celebrated motto of the zoologist Theodosius Dobzhansky is particularly true in developmental biology and in cancer, that is firstly an evolutionary disease, more precisely a disease of evolutionary multicellular organisation because it may be considered as a backward step in the course of evolution towards organised multicellularity, according to the atavistic theory of cancer ([5], [12], see next Subsection). Cancer thus represents an evolution at the time scale of a living multicellular organism, anatomically localised in a given organ, possibly extended to other tissues by remote metastases, towards genetically new unicellular species developing, likely with branching by successions of mutations, their diversity at the expense of the host organism. I contend that the disappearance of successive physiological control mechanisms puts cancer cell populations in the state of a very primitive multicellular organisation, as proposed below.

\subsection{The atavistic theory of cancer}

In 2011, independently, physicists Paul Davies and Charles Lineweaver, and oncologist Mark Vincent avocated the idea that cancer is a de-repression of a default survival program common to all cells [12] which was expressed by Davies and Lineweaver as the atavistic theory of cancer [5]. Alternatively saying, cancer is a disease of the evolution of multicellular organisms in which a localised collection of cells organises itself and proliferates for its own benefit (possibly trying to reinvent the wheel of multicellularity and bound to failure). This hypothesis has been assessed from phylostratigraphic analyses of the genomes of different species that allowed to establish links between the genes that are essential to multicellularity and those that are altered in cancer, and from more recent studies that elicit disrupted relationships between genes of multicellularity and genes that are disrupted in cancer. This emerging field of research of course presupposes that cancer is an evolutionary disease of multicellular organisms, 'evolutionary' here meaning related to the Darwinian evolution of living species. In this respect, 
plasticity is related to loss of control of the differentiations that make a multicellular organism coherent and functional, and any disruption in this control may lead to cancer, without necessarily resorting to cancer stem cells. In other words, loss of control on differentiation, which is most likely related to defective control by the immune system, is actually the plasticity of cancer.

\subsection{Failed control of differentiations: cancer is a failure of cohesion}

The task of the hypothesised cohesion watch, part of an extended version of the immune system, is thus to ensure compatibilities a) between morphogens of the body plan, able to drive it actually from the zygote to a constituted multicellular being in an irreversible way within the 3D space of cells of a given individual (in jawed vertebrates defined by the major hiostocompatibility complex, MHC, and by some likely equivalent forerunners in non-vertebrates); b) between phenotypic functionalities, ensuring compatibility between differentiation trees that yield lineages within a given subpopulation during embryogenesis, and ultimately between cooperating subpopulations (division of work) of terminally differentiated cells; c) between the body plan space distribution and the time distribution of phenotypes in each epigenetic landscape attached to the body plan.

To mentally illustrate this construction, I propose as a further metaphor the wickerwork basket. Starting from a circle endowed with lots of connections between its elements, that is supposed to represent the body plan, functional willow-like twigs stem from each of these elements, representing the great physiological functions of the organism. If no weaving is made between these twigs, the whole set will consist of just flexible differentiation functionalities of a family of cell types, floating freely in the surrounding space, unrelated to each other. No cohesion, no division of labour can result from such unwoven twigs and trees. The task of the cohesion watch is to ensure such weaving during development, along the twigs from stem cells until tips that are terminally differentiated cells. This naturally includes the solidity of the willow twigs (breaches along the vertical axis resulting in blocked differentiations), but the main part of the cohesion watch is to ensure compatibility between neighbouring twigs.

\subsection{Speculations on the possible future of cancer therapeutics}

Rather than fighting uncontrolled proliferation, could we repair altered control on differentiations? Cell-killing strategies, be they relying on chemotherapies or on modern immune cell-enhancing drugs, miss the basic targets, that are differentiation sites, and better work would undoubtedly be done by enforcing connections ensured by the cohesion watch, rather than by killing cheater cells. Or else, trying to illustrate this goal with a sociological metaphor: rather than killing cheater cells by cannonade (i.e., by chemotherapies) or by enforcing the aggressiveness of the police (i.e., by immune checkpoint inhibitors), would it not be better to imagine how to enforce natural cohesion between cells? To be able to do this, a better understanding of the mechanisms of control of differentiation at the level of local transcription factors and at the level of chromatin is needed. 


\section{Conclusion}

Far from considerations on evolution of a cell population at the time scale of a human life - my starting point [4], [6] - , that undoubtedly present a high interest in therapeutics, I have presented in this study an evolutionary point of view on cancer in a billion-year perspective that, from questions on plasticity in cancer, drove me to develop ideas and speculations that resort to what is now known as philosophy of cancer [1], [8], [9], thus beginning to tread a long and winding path towards a fundamental understanding of multicellularity and of its alterations in cancer, that should lead to correct impaired control of differentiation, rather than, or at least together with, control of proliferation.

\section{References}

1. Bertolaso, M.: 'Philosophy of cancer. A dynamic and relational view'. Springer, Heidelberg, New York (2016)

2. Chisholm, R.H., Lorenzi, T. \&Clairambault, J.: Cell population heterogeneity and evolution towards drug resistance in cancer: Biological and mathematical assessment, theoretical treatment optimisation. Biochim Biophys Acta, 1860 2627-2645 https://doi.org/10.1016/j.bbagen.2016.06.009 (2016)

3. Clairambault, J.: Stepping from modelling cancer plasticity to philosophy of cancer. Frontiers in Genetics, accepted https://doi.org/10.3389/fgene.2020.579738 (2020)

4. Clairambault, J. \& Pouchol, C.: A survey of adaptive cell population dynamics models of emergence of drug resistance in cancer, and open questions about evolution and cancer. Biomath 8:1905147, 23 pages, https://doi.org/10.11145/j.biomath.2019.05.147 (2019)

5. Davies, P.C.W. \& Lineweaver, C.H.: Cancer tumors as Metazoa 1.0: tapping genes of ancient ancestors. Phys. Biol. 8(1):015001, 7 pages, https://doi.org/10.1088/1478-3975/8/1/015001 (2011)

6. Pouchol, C., Clairambault, J., Lorz, A. \& Trélat, E., Asymptotic analysis and optimal control of an integro-differential system modelling healthy and cancer cells exposed to chemotherapy. J. Math. Pures Appl. 116, 268-308 https://doi.org/10.1016/j.matpur.2017.10.007 (2018)

7. Lineweaver, C.H., Davies, P.C.W. \&Vincent, M.: Targeting cancers weaknesses (not its strengths): Therapeutic strategies suggested by the atavistic model. BioEssays 36:827835 https://doi.org/10.1002/bies.201400070 (2014)

8. Pradeu, T.: 'The limits of the Self'. Oxford University Press (2012)

9. Pradeu, T.: 'Philosophy of immunology'. Cambridge University Press (2019)

10. Sharma, S.V., et al.: A Chromatin-Mediated Reversible DrugTolerant State in Cancer Cell Subpopulations. Cell 141, 69-80 https://doi.org/10.1016/j.cell.2010.02.027 (2010)

11. Shen, S. \& Clairambault, J.: Cell plasticity in cancer cell populations (review) [version 1; peer review: 2 approved]. F1000Research 2020, 9 (F1000 Faculty Rev) 635 , 16 pages, https://doi.org/10.12688/f1000research.24803.1 (2020)

12. Vincent, M.D.: Cancer: a de-repression of a default survival program common to all cells?: a life-history perspective on the nature of cancer. Bioessays 34(1), 72-82 https://doi.org/10.1002/bies.201100049 (2011) 